\title{
INSERTION PROFESSIONNELLE D'IMMIGRANTS RÉCENTS ET RÉSEAUX SOCIAUX: LE CAS DE Maghrébins À Montréal et SHERBROOKE ${ }^{1}$
}

\author{
SÉBASTIEN ARCAND \\ ANNICK LENOIR-ACHDJIAN \\ Denise Helly
}

Résumé. Afin de mieux comprendre les représentations que donnent des acteurs d'origine maghrébine des difficultés qu'ils rencontrent à insérer le marché de l'emploi québécois, nous avons mené entre 2001 et 2004 des entrevues auprès de 22 Maghrébins en recherche d'emploi à Montréal et Sherbrooke. Des entrevues ont aussi été menées avec 15 intervenants d'organismes gouvernementaux œuvrant auprès d'une clientèle en recherche d'emploi, dont des Maghrébins. Dans cet article, l'objectif est de saisir à travers les trajectoires individuelles et collectives l'évolution et l'usage des réseaux de ces immigrants. Nous verrons notamment que la présence d'une communauté de même origine ethnoculturelle ne peut garantir l'élargissement des réseaux s'il n'y a pas de soutien institutionnel significatif et ce, tant du côté de la société que de la communauté elle-même. Mots-clés: réseaux sociaux, maghrébins, intégration, marché de l'emploi, Québec

Abstract. Between 2001-2004 we conducted 22 interviews with immigrants from North Africa in Montreal and Sherbrooke. The objective was to access their representations of the problems they experience in integrating the labor market in both cities. Some interviews (15) were also conducted with persons working with immigrants looking for a job. The objective is to link individual and collective trajectories by putting the emphasis on the social networks held by these immigrants. Results show that the presence of a community of origin in a migratory context cannot guarantee access to a viable social network providing access to information and jobs. In that sense institutional supports and programs may favor an increase of social networks.

Keywords: social networks, North Africans, integration, labour market, Quebec

1. Cette recherche a bénéficié de fonds de recherche du Conseil de recherche en sciences sociales du Canada (CRSH) et de Patrimoine Canada dans le cadre du concours «Questions de multiculturalisme». 
J'ai beaucoup de connaissances, mais je ne sais pas si ça va m'aider. Si mon réseau va m'aider à trouver de l'emploi ou pas. Ça je l'ignore, parce que des amis c'est un réseau, pour l'emploi je ne sais pas.

Un nouvel arrivant d'origine maghrébine

I 'insertion socio-économique des nouveaux arrivants est détermi_née par les contextes des sociétés d'immigration et par les pratiques collectives et individuelles pour surmonter les barrières structurelles et systémiques. Dans le contexte canadien, quelque $60 \%$ des immigrants adultes arrivés depuis 2000 détiennent un diplôme post-secondaire comparativement à $40 \%$ de l'ensemble des Canadiens et $35.7 \%$ des migrants arrivés entre 1996-2001 détenaient un diplôme universitaire comparativement à $13.8 \%$ des natifs. En dépit de ce haut niveau de scolarité et d'une sélection plus sévère depuis 2002, le niveau des salaires de ces immigrants est insatisfaisant (Picot 2004; Sweetman 2006) et leur taux de chômage élevé. En 2006 ce taux au Canada était de 5.5\% et selon une étude de 650000 immigrés (Statistique Canada 2005; 2006) de 11\% pour les immigrants des années 2001-2006, oscillant selon la région: Asie $11 \%$, d'Amérique latine 10.5\%, d'Europe 8.4\%, d'Afrique 20.8\%. Seuls les Philippins avaient le même taux que les natifs, ils disposent d'un réseau communautaire d'aide établi de longue date et $80 \%$ ont un diplôme post-secondaire et parlent anglais à l'arrivée. À l'opposé les immigrés africains montraient de nettes difficultés; $20 \%$ sont des réfugiés qui faute de documents ne s'insèrent pas sur le marché de l'emploi rapidement; ils composent de nouveaux courants migratoires et ne disposent pas de réseau communautaire local d'aide et d'emploi. Par ailleurs le taux de chômage des immigrants des années 1996-2001 s'élevait à 7.3\% et de ceux établis avant 1996 à 5.5\% . La situation était aussi négative au Québec. En 2006, le taux de chômage provincial était 6\% et celui des immigrés arrivés avant $19969 \%$ mais s'élevait à $13.4 \%$ pour les immigrants de 1996 à 2001 et $18.7 \%$ pour les immigrants de 2001 à 2006 (Afrique 27.1\%, Amérique latine 15.4\%, Asie 13.3\%, Europe 13.2\%).

Selon les données de Statistique Canada, en 2000, les hommes immigrés obtenaient un salaire de 63,1 cents versus $1 \$$ par les natifs de même niveau scolaire ${ }^{2}$ alors que ce rapport était de 71,6 cents en 1980 pour les hommes arrivés cette année-là. De plus, les hommes immigrés depuis dix ans recevaient en moyenne 79,8 cents comparativement à $1 \$$ par les natifs détenant le même niveau de scolarité; ce rapport était égal en 1980 (1\$ pour tous). Enfin, jusqu'au début des années 1980, les immigrés rattrapaient en dix ans le handicap de ne pas détenir d'expérience de travail

2. $40 \%$ des immigrés des années 1990 âgés de 25 à 54 ans détenaient un diplôme universitaire versus $23 \%$ des natifs de même âge. 
canadienne. La situation des immigrés arrivés depuis les années 1980, dont une fraction importante est musulmane, apparaît donc défavorable.

Diverses raisons sont à la source de cette faible performance: élimination d'emplois manufacturiers occupés par les immigrés peu qualifiés, souvent parrainés (5\% des nouveaux postes de travail exigent une scolarité de secondaire, $70 \%$ une scolarité post-secondaire); exigence d'une maitrise élevée du français ou de l'anglais dans les nouveaux emplois qualifiés; hausse de la scolarité des natifs; difficulté ou refus des autorités, corps de métier et corporations d'estimer la valeur des qualifications acquises à l'étranger mais non garanties par un diplôme; en dépit de la Loi d'Équité en emploi et de Embracing Change Initiative pour recruter des minorités visibles (2000), faible absorption par la fonction publique fédérale d'une proportion significative de la main d'œuvre immigrante vu le pré requis du bilinguisme pour nombre de postes; discrimination à l'emploi (Helly, Nong Zhu, et Trudel 2008).

Dans ce contexte plutôt difficile, il est envisageable que, une fois installés dans leur nouveau pays de résidence, les immigrants usent de diverses stratégies d'insertion en emploi pour accéder au marché du travail ou pour favoriser leur mobilité socio-occupationnelle. Une étude de Statistique Canada publiée en 2005, ELIC, indique que la stratégie la plus suivie par les nouveaux arrivants lors de la quête d'un premier emploi est d'entrer directement en communication avec l'employeur (90\%) alors que le recours aux parents et amis ainsi qu'aux bureaux d'emploi est utilisé dans $44 \%$ des cas. Ces données portent à questionner le rôle des liens que tissent les nouveaux arrivants lors de leur quête d'un emploi. Pour pallier aux lacunes des recherches existantes et compte tenu des politiques migratoires qui favorisent la venue d'immigrants jeunes et qualifiés, il y a lieu de s'attarder à cette dernière catégorie de personnes et à l'apport des réseaux sociaux dans son insertion professionnelle.

La majorité des études mettent de l'avant les difficultés d'intégration en s'intéressant soit à des facteurs structurels (Reitz 2005; Frenette et Morissette 2003), soit au rôle des organismes d'aide à l'emploi et des associations ethniques, une forme de réseaux distincte de celle qui nous intéresse, soit encore aux qualifications des migrants (Lo 2003; Renaud et al. 2003; Piché et al. 2002; Préfontaine et Benson 1999). L'approche par le réseau social permet de voir comment des individus vivant des expériences similaires développent des pratiques différentes pour faire face à leurs difficultés d'intégration. L'objectif du présent article est de saisir la portée des réseaux de travailleurs qualifiés d'origine maghrébine installés au Québec depuis moins de trois ans (en 2004) et en recherche d'un emploi en lien avec leurs qualifications. Le cadre conceptuel et théorique s'inspire des travaux de Granovetter $(1973,1985,1990)$ pour 
mieux comprendre l'influence des réseaux sur l'accès à un emploi qualifié à partir d'une analyse des «liens forts» (famille, amis) et des «liens faibles» (contacts professionnels, institutionnels) que possède un groupe donné ou un individu.

Plusieurs raisons sont à l'origine de cet intérêt pour des professionnels maghrébins récemment installés au Québec. On observe un accroissement significatif de l'immigration en provenance du Maghreb au Québec depuis les dix dernières années (Institut de la statistique du Québec 2007), une immigration dont la capacité à s'intégrer rapidement est dite prometteuse. On observe, comme pour l'ensemble des nouveaux arrivants, une dégradation continue de la situation économique des Maghrébins (Bauder 2001; Germain et al. 2001; Renaud et al. 2003), particulièrement depuis le 11 septembre 2001. ${ }^{3}$ Pour mettre en contexte l'apport des réseaux sociaux des personnes interrogées, cet article met l'accent sur la dynamique entre les pratiques de l'État (sélection et programmes d'insertion en emploi), le contexte sociétal (difficultés d'emploi) et les stratégies des immigrants, dont le recours à des réseaux. Plus particulièrement, il s'intéresse aux liens intra et extra communautaires en contexte post migratoire. Cela n'exclue pas la prise en compte du contexte pré migratoire présentée en introduction de l'analyse. Les raisons de ces choix sont doubles. Premièrement, porter attention aux possibilités de nouveaux arrivants d'origine maghrébine d'accéder à un réseau de contacts professionnels utile à l'obtention d'un emploi en lien avec les qualifications et le niveau d'éducation pour lequel ils ont été sélectionnés. Deuxièmement, identifier les possibilités que donne la sociologie des réseaux sociaux de mieux saisir les processus à l'œuvre dans la construction/destruction des relations sociales lors du temps migratoire. ${ }^{4}$

\section{DE LA NOTION DE RÉSEAU SOCIAL}

À l'instar de nombreux autres champs théoriques, celui des réseaux sociaux est balayé par une pluralité d'approches et de méthodologies

3. Certains aspects de cette problématique ont d'ailleurs été traités par Lenoir-Achdjian et al. (2008)

4. Par «temps migratoire», nous entendons l'ensemble du processus qui concerne les différentes étapes d'insertion socio-économique des immigrants. Cela comprend autant les dimensions matérielles (un endroit pour vivre, un travail, une école pour les enfants, etc.) que sociales (les fréquentations, les activités politiques et civiques, etc.). Ce temps migratoire n'étant pas le propre de nouveaux arrivants mais de l'ensemble des immigrants, il s'agit plutôt de poser un regard sociohistorique, même avec très peu de recul comme dans le cas présent, sur la dynamique d'insertion. Inspirée du concept de «cycle migratoire» développé par les théoriciens de l'École de Chicago dans les années 1920-30, la notion de temps migratoire s'en démarque néanmoins par une lecture moins «économiciste» des relations sociales. 
(Mercklé 2004). C'est en fonction de cette pluralité de perspectives que nous tracerons les balises théoriques de la recherche. Depuis plusieurs décennies les théories des réseaux sociaux ne cessent de se développer au point de devenir un élément explicatif important de l'étude des sociétés contemporaines, notamment parce qu'elles permettent de recréer le lien entre société et individu. Dans ce champ certains auteurs sont incontournables.

C'est le cas de M. Granovetter $(1973 ; 1990)$ qui a conceptualisé la formation des réseaux et a mis en évidence leur importance dans la structuration des rapports sociaux contemporains. Ses travaux demeurent d'une grande actualité pour les différents aspects liés à la question de l'emploi. La théorie des réseaux sociaux de Granovetter dépasse la rigidité des concepts traditionnels en sociologie tels que la classe sociale, la communauté et la société. ${ }^{5}$ Plus important encore, elle considère généralement que les actions des acteurs sont déterminées par l'organisation sociale et que les liens qu'ils tissent sont conditionnés par des éléments extérieurs à leur propre volonté. Cela signifie que l'on ne peut en appeler qu'à l'initiative personnelle et faire reposer le fardeau de l'insertion socioprofessionnelle sur les seules épaules des nouveaux arrivants puisque le contexte local fournit à la fois les ouvertures et les barrières à cette insertion.

Le concept de réseau social fait référence à la nature des «liens faibles» et des «liens forts», des liens conditionnés par une hiérarchisation des relations entre acteurs institutionnels et individuels. Les liens forts marquent un haut degré de ressemblance entre deux ou plusieurs personnes (famille, amis). Ils ne relient pas entre eux des groupes dissemblables et une information qui circule uniquement à travers des liens forts risque de rester circonscrite à un groupe restreint. À l'opposé, les liens faibles favorisent la circulation des individus et des groupes dans un réseau plus vaste ou de «clique en clique» (Granovetter 1973). Pour le chercheur d'emploi, il est important d'élargir ses réseaux pour accéder à l'information relative aux emplois disponibles. Par conséquent la sociologie des réseaux sociaux, celle de Granovetter mais aussi de Freeman (1979), Coleman (1990), Boudon (1988) et autres auteurs est fort utile pour montrer l'importance des réseaux, principalement des liens faibles, pour les nouveaux arrivants lors de leur recherche d'emploi. Quand dé-

5. Nous ne remettons pas en cause la pertinence d'une lecture des problèmes d'insertion socio-économiques des immigrants et des minorités en général à partir des classes sociales telle que, par exemple, Bonacich $(1973 ; 1976)$ l'a exprimé en termes clairs. Cela dit, il apparaît que conceptualiser l'idée même d'un groupe, qu'il s'agisse de classe, de groupe ethnique ou de catégorie professionnelle, comme étant homogène ne favorise pas une compréhension des liens que tissent, ou ne tissent pas, les acteurs sociaux dans un contexte migratoire. 
veloppé, le réseau social constitue pour un individu un capital social à partir duquel il peut 'négocier' son entrée sur le marché de l'emploi. ${ }^{6} \mathrm{Au}$ cours des dernières décennies, plusieurs recherches sur la thématique des réseaux sociaux des immigrants ont été réalisées et sont fort instructives pour la présente recherche.

\section{LA REVUE DE LITTÉRATURE}

Wilson et Portes (1980) montrent l'existence d'enclaves ethniques à partir desquelles des immigrants récents tentent de s'incorporer à la vie socio économique de leur pays d'adoption. Portant sur la communauté cubaine de Miami, cette étude apporte des éléments sur le développement des réseaux sociaux et Les opportunités et degré d'ouverture des économies capitalistes avancées. Toutefois, elle diffère de la nôtre parce que les Maghrébins interrogés au Québec fréquentaient des Centres locaux d'emploi au moment de l'enquête. Ils ne cherchaient pas nécessairement à s'insérer au sein d'une enclave ethnique mais plutôt dans un marché général de l'emploi. Cette caractéristique rejoint la situation mentionnée par Portes et Manning (1986): les immigrants qualifiés s'insèrent dans la «Primary Economy» et avec le temps accèdent aux mêmes échelons occupationnels que les natifs. Dans des contextes autres que le Québec, des études récentes se sont intéressées à la problématique des réseaux sociaux des nouveaux arrivants et à leur impact sur l'insertion socio-économique. C'est le cas notamment de Molina et McCarty (2007) qui se penchent sur les liens entre l'auto-identification ethnique et les réseaux personnels d'immigrants en Espagne. Dans un autre contexte, et plus directement sur notre thème d'investigation, Amuedo-Dorantes et Mundra (2007) examinent l'influence de différents types de réseaux sociaux sur les salaires gagnés par les migrants mexicains légaux et illégaux aux États-Unis. Toujours à propos de la population mexicaine aux États-Unis, Garcia (2005) montre qu'au-delà de l'idée d'un réseau dit «ethnique» se trouve un ensemble de sous-réseaux (traditionnel, église et un réseau de petits contrats) qui fournit des services et favorise l'élargissement, la force et la densité de la communauté immigrante locale. Pour leur part, Iosifides et al. (2007) s'intéressent au rôle du capital social dans les différentes formes (sociale, économique, institutionnelle)

6. La relation entre le réseau social et le capital social tient à la capacité et aux possibilités pour les individus d'user de certaines compétences (qualifications, expériences professionnelles, compétences linguistiques, etc.) pour accéder à de l'information spécifique sur des offres d'emplois, voire directement à des emplois. Pour plus de détails sur la notion de capital social, voir Coleman (1990), Bourdieu (1980), Loury (1977), Putnam (2001; 2007), Woolcock (2001). 
d'incorporation d'Albanais dans la société grecque. Les auteurs identifient trois formes de capital social, le 'lien' (bonding), le 'relais' (bridging) et les 'connections' (linking).

Pour leur part Fuglerud et Engebrigtsen (2006), dans une recherche sur les Somaliens et les Tamouls en Norvège, en appellent à une redéfinition des notions de capital social et de réseaux sociaux en y incorporant la dimension transnationale via le concept de culture. La recherche de Fuglerud et Engebrigtsen est inspirante car elle montre l'importance des liens qu'entretiennent les nouveaux arrivants avec leur pays d'origine. Dans le contexte canadien, Kazemipur (2006) s'est intéressé à la place qu'occupe le capital social au sein des dynamiques migratoires internationales, allant de l'idée de quitter le pays d'origine au succès ou échec de l'installation dans le pays d'immigration. Parmi les résultats obtenus via un sondage effectué auprès de plus de 600 personnes, immigrants comme non-immigrants, Kazemipur conclut que les réseaux sociaux des immigrants, contrairement à ce que avancent certaines études américaines, sont «moins importants que ceux des individus nés au Canada» et moins rentables ce qui, comme nous le verrons, rejoint en partie nos conclusions de recherche. Toujours dans le contexte canadien mais cette fois dans une perspective comparative avec les États-Unis et auprès d'immigrants d'origine brésilienne, Goza (2003) étudie les manières dont les réseaux affectent différents aspects de l'immigration et de l'intégration (décision de quitter son pays, pays de destination, adaptation dans le pays d'origine, ainsi que les effets que de tels réseaux peuvent avoir sur les salaires futurs). À l'instar du travail de d'Amuedo-Dorantes et Mundra (2007) mentionné précédemment, l'étude de Goza permet de prendre la mesure des salaires obtenus par les immigrants en fonction de la force de leurs réseaux sociaux respectifs. Sur le plan du choix de l'immigration et du pays de destination, l'article de Goza rejoint la recherche de Collyer (2005) qui, à partir d'une population similaire à celle qui nous occupe, examine les raisons qui poussent les réfugiés algériens en Europe à privilégier un pays plutôt qu'un autre. Collyer conclut que les sans-papiers ont tendance à mettre l'accent sur leurs liens faibles plus que sur les réseaux familiaux dans le choix du pays de destination et lors de leur insertion dans celui-ci. Mentionnons enfin que selon une enquête de Statistique Canada (2004), 98\% des immigrants installés au Canada n'ont pas fait de demande dans un autre pays avant de venir s'établir au Canada. De plus, dans 27\% des cas les personnes interrogées par Statistique Canada ont affirmé être venues au Canada pour rejoindre un ou des membres de leur famille.

Plusieurs études récentes ont ainsi été effectuées auprès de groupes et dans des contextes différents. Elles permettent de comprendre l'influence des réseaux sociaux sur le processus migratoire et d'installation 
des nouveaux arrivants. Toutefois, aucune ne porte spécifiquement sur le contexte québécois et très peu mettent l'accent sur la recherche d'emploi en lien avec les compétences professionnelles des nouveaux arrivants issus de la catégorie des travailleurs qualifiés; c'est ce que nous nous proposons de faire

\section{Délimitations de la ReCherche et élÉMENTS de MÉTHOdOLOGIE}

Dans cette recherche spécifique, nous avons tenté de valider ou d'invalider un ensemble d'affirmations issues de recherches antérieures et de voir si elles s'appliqueraient aux Maghrébins. La question de recherche se formule comme suit: Les réseaux sociaux des Maghrébins en situation post migratoire au Québec favorisent-ils leur insertion sur le marché de l'emploi? Cette question appelle quatre propositions issues de la littérature et une hypothèse. Selon Bergeron et Potter (2006), dans une étude sur les femmes immigrantes, la présence de membres d'une même communauté d'origine favorise l'obtention d'un emploi, en lien ou non avec la profession. Nous reprenons cette proposition tout en ne tenant pas compte de la distinction de genre. Par la suite, nous nous intéressons au lien possible entre la fréquentation d'associations maghrébines (Association des Marocains du Québec, Forum musulman canadien, etc.) et l'accès à un emploi. Certaines études abordent la question des associations et de l'élargissement possible des réseaux sociaux mais les conclusions sur cette question sont mitigées comme nous l'enseignent Paré (2002) et Cardinal et Tremblay (1998) sur les entrepreneurs ou encore Potocky-Tripodi (2004) et Li (2004) sur la question du capital social, des associations et de la mobilité socio-économique. Pour ouvrir la réflexion à des acteurs extérieurs au groupe étudié, notre troisième proposition s'intéresse au travail des organismes gouvernementaux d'aide à l'emploi et d'intégration et à son impact sur l'obtention d'un emploi (cf. Gouvernement du Québec 2004a; 2004b; 2004c; Gouvernement du Canada 2000). Notre quatrième proposition est liée aux études qui indiquent que l'obtention d'un premier emploi par des personnes qualifiées permet à moyen terme de connaître une mobilité socio-économique. Cette proposition rejoint l'idée de Portes et Manning (1986) à propos des travailleurs qualifiés qui s'insèrent dans des secteurs d'activité économiques dominés par les natifs. Finalement, nous émettons l'hypothèse que la faiblesse des réseaux sociaux des Maghrébins récemment installés au Québec favorise, à court et moyen terme, une recherche individuelle de solutions conduisant à une désillusion et un désengagement à l'égard du marché de l'emploi. 


\subsection{La collecte des données}

Le corpus est composé de Maghrébins professionnels âgés de 25 à 40 ans, arrivés au Québec depuis trois ans ou moins au moment de l'enquête et en recherche d'emploi (2004-05). L'âge et la région de provenance des répondants sont liés aux objectifs de la politique d'immigration du Québec en vigueur entre 1996 et 2005 qui sont de favoriser la venue de jeunes adultes, idéalement des familles ou des couples, détenant minimalement un diplôme d'études post-secondaires et ayant une bonne connaissance du français. Ainsi, les répondants constituent une immigration prometteuse, voire idéale, car elle doit permettre de pallier certains problèmes démographiques, linguistiques et économiques auxquels fait face le Québec contemporain (Vatz-Laaroussi 2008). Le contact avec les répondants potentiels a été possible grâce à l'aide d'organismes gouvernementaux tels que le ministère de l'Immigration et des Communautés Culturelles, les Centres locaux d'emploi et des organismes d'insertion en emploi subventionnés par Emploi-Québec. Au total, 22 entrevues ont été réalisées avec des Maghrébins (17 hommes et 5 femmes) habitant à Montréal (16 personnes) ou Sherbrooke (6 personnes), de même qu'avec quinze (15) intervenants d'organismes œuvrant auprès de cette clientèle à Montréal ( 8 personnes) et à Sherbrooke ( 7 personnes). Considérant que plus de $80 \%$ des immigrants au Québec s'installent dans la région montréalaise, le choix de la métropole québécoise s'imposait (67 830 maghrébins en 2001). Quant à Sherbrooke, il s'agit d'une région prisée par le gouvernement dans sa politique de régionalisation de l'immigration et en 2001 il y eut 420 personnes de langue maternelle arabe, dont 120 marocains (les chiffres sur les Algériens ne sont pas disponibles) (Statistique Canada, 2008). La comparaison entre ces deux villes permettra de mettre en parallèle deux contextes d'installation, l'un hautement multiculturel (Montréal), l'autre situé en périphérie et de plus petite taille.

Avec l'aide du logiciel Atlas-Ti, nous avons procédé à une analyse de contenu pour éliminer les tentations de la sociologie «spontanée» en recherchant ce que Gardin (1974) nomme le «dépassement de l'incertitude et l'enrichissement de la lecture». Lorsque la collecte de données est de type «entretien semi directif» comme dans notre cas, elle exige un équilibre entre laisser parler les gens de leur trajectoire, de leur histoire, voire de leur frustration de ne pas trouver un emploi, et l'orientation des entrevues de manière à demeurer dans le sillon tracé par la question de recherche (Gauthier 2009; d'Unrug 1974). ${ }^{7}$ Dans notre cas l'idée, très

7. La question de la validité de l'analyse qualitative soulève régulièrement un débat entre chercheurs, notamment du fait qu'elle est en général adoptée pour traiter de petits échantillons. Mais, en accord avec Andréani et Conchon (2003), de même qu'avec Mucchielli (1996), nous croyons que l'analyse qualitative est la plus adéquate lorsque le but cher- 
wébérienne, de reconnaître la rationalité du discours des Maghrébins fait en sorte que les données obtenues sont considérées comme un processus de rationalisation et d'objectivation des acteurs à l'égard de leur propre condition de migrants.

\subsection{De la codification à l'analyse : une démarche exploratoire}

Pour l'analyse nous avons codifié les passages retenus en fonction de leur référence à la thématique des réseaux sociaux. Par la suite, nous avons mis en relation ces codifications pour en arriver à une deuxième codification plus complexe et plus apte à faire ressortir l'évolution des réseaux sociaux au fil du temps. Puisque les compétences - scolarité, âge, connaissance du français - sont largement similaires d'une personne à l'autre, nous avons segmenté les entrevues en fonction de ces compétences et caractéristiques $\left(1^{\mathrm{er}}\right.$ segment $=$ niveau d'éducation, $2^{\mathrm{e}}$ segment $\left.=\hat{\mathrm{a} g e}\right)$. Cela a permis d'identifier le système de réseaux des Maghrébins interrogés et d'évaluer leurs possibilités d'accéder à des contacts pouvant faciliter l'obtention d'une entrevue d'emploi ou d'un emploi.

Les liens entre les codes se sont faits en fonction d'adéquations significatives (liens de causalité non déterministes) que peuvent entretenir des passages d'entrevues. Par exemple, si une personne souligne qu'elle est venue s'installer à Montréal dans le but d'améliorer sa situation financière et qu'ensuite elle s'identifie plus comme un ingénieur qu'un Algérien, la volonté d'améliorer la situation financière et le désir de se trouver un emploi dans le domaine de l'ingénierie sont liés. Ce lien peut être transposé à d'autres segments d'entrevue faisant état de la difficulté à entrer en contact avec des Québécois d'origine canadienne française. Nous avons procédé ainsi pour chacune des 22 entrevues avec des Maghrébins. Les entrevues avec les intervenants ont servi à corroborer ou atténuer certaines affirmations. Par la suite nous avons mis en relation ces entrevues en fonction des adéquations significatives identifiées.

\section{Faire le saut! Comment des liens en Situation de pré migration PEUVENT INFLUENCER L'INSERTION POST MIGRATOIRE}

Rappelons que l'analyse cherche à valider ou invalider les propositions présentées précédemment. Ainsi le réseau pré migratoire, c'est-à-dire les relations du futur migrant avec des proches (parents, amis), des profes-

ché, comme dans le cas de cette étude, est d'explorer les pensées, les rites, le sens symbolique et les représentations des répondants, bref de saisir la réalité dans sa complexité. Parce que la présence de critères validant les résultats demeure essentielle, la validité interprétative (réflexivité, introspection, interaction et triangulation avec des études similaires) et la validité communicationnelle (accord entre les répondants, généralisation potentielle des résultats) peuvent faire office de balises (Andréani et Conchon 2003). 
sionnels spécialistes des lois d'immigration résidant dans le pays d'origine et avec les instances du futur pays d'immigration (fonctionnaires des délégations du Québec et des ambassades canadiennes), joue un rôle déterminant dans le processus migratoire. Ces personnes ont une influence sur un ensemble d'éléments allant de la décision de partir en passant par l'ensemble des formalités administratives que tout futur immigrant doit remplir. À titre d'exemple, des fonctionnaires recevant des candidats à l'immigration peuvent, comme rapporté par des personnes interrogées, mentionner aux candidats qu'ils ont tous les atouts pour être sélectionnés et pour s'intégrer adéquatement au marché de l'emploi québécois. Cela dit, on doit distinguer le réseau intra communautaire du réseau extra communautaire car ces réseaux n'ont pas le même impact sur le processus d'insertion des Maghrébins une fois installés au Québec.

\subsection{Liens forts et liens faibles : un renforcement positif}

En ce qui concerne le réseau intra communautaire, il est souvent à l'origine de la décision d'immigrer au Canada et plus spécifiquement au Québec que ce soit à Montréal ou À Sherbrooke. Le Canada et le Québec sont généralement dépeints sous un angle très positif par les personnes du réseau intra communautaire situé dans les pays d'origine (Maroc ou Algérie):

J'ai des amis qui sont venus ici à Québec avant, on a fait les études au Maroc ensemble, on a passé une partie du travail ensemble au Maroc. Ils ont quitté le pays, ils sont venus ici avec leurs familles. Il y en a qui passent une partie de l'été et qui reviennent. Il y en a qui m’ont parlé du Québec, ils sont très satisfaits, alors je suis venu. (migrant \#7)

Le niveau de satisfaction exprimé par ce réseau intra communautaire sur la vie au Québec devient un facteur déterminant de la décision de migrer vers cette province. Le réseau de liens forts dans un contexte pré migratoire ne doit pas être interprété comme un facteur clé de l'émigration mais comme un facteur renforçant positivement la décision de partir. De fait, dans les cas où les échos du Québec sont négatifs, ces liens forts n'ont aucune portée significative lors de la pré migration et ils n'hypothèquent pas la décision de partir :

Avant de venir, j'ai rencontré un Marocain qui était là. Il est revenu au Maroc. Là, il a fait une affaire au Maroc, il est resté au Maroc. Avant de venir je l'ai rencontré, il m'a déçu. Il m'a dit, 'Si tu veux aller au Canada, il y a rien là-bas'. Personnellement je ne l'ai pas crû. J'ai dit bon, c'est quelqu'un qui n'est pas efficace. C'est pour ça qu'il me dit ça. Mais finalement je me suis aperçu que c'est pratiquement ça. (migrant \#1)

Cette question des échos positifs et négatifs en situation pré migratoire doit être interprétée en relation avec l'image perçue et véhiculée 
du Québec à l'étranger. Le Québec est généralement considéré comme un endroit où l'on peut accéder à un certain «rêve américain», c'est-àdire une mobilité sociale, une plus grande liberté d'action et de pensée, etc., tout en communiquant en français. ${ }^{8}$ Le facteur linguistique devrait, pensent les candidats, constituer un avantage en leur faveur compte tenu de leur connaissance préalable du français et de leur niveau de scolarité élevé. Cependant, après la migration, il arrive que les discours se fassent plus critiques. Les personnes interrogées considèrent en effet qu'elles n'ont pas été suffisamment informées des obstacles potentiels à leur insertion en emploi. Cette perception d'avoir été trompés, partagée par une majorité de répondants, n'est toutefois pas corroborée par les intervenants, même si une partie de ces derniers disent se questionner sur ce qui se dit vraiment à l'étranger :

Ils veulent faire rentrer, rentrer, rentrer des gens sans vraiment réfléchir aux conséquences chez ces personnes-là qui vont immigrer. Alors qu'ils arrêtent de dire, dans ces pays-là, qu'il y a beaucoup de travail et que vous allez faire une carrière ici au Québec quand c'est difficile. (intervenant \#6)

Cette citation illustre les problèmes de concordance entre les discours des organismes en charge de recruter, sélectionner ou d'intégrer les immigrants. On notera par ailleurs que l'on véhicule du côté des intervenants une vision positive du rôle de la communauté d'origine en contexte post migratoire :

La communauté est suffisamment organisée d'après moi pour qu'ils puissent avoir dans leur milieu de bons contacts. Quand ils arrivent, j'ose croire qu'ils prennent contact avec la communauté maghrébine ou les Haïtiens avec les Haïtiens ... puis l'information circule dans les amis, les collègues qu'ils vont se faire, qu'ils vont connaitre. (intervenant \#5)

Comme nous le verrons, les immigrants interrogés ne partagent pas cette idée sur leur communauté et surtout de l'aide qu'ils peuvent en obtenir lors de leur recherche d'emploi.

\subsection{Distorsions discursives et idéalisation de l'immigration}

Outre certains professionnels, souvent des avocats locaux, qui se spécialisent dans la préparation des dossiers d'immigration, le réseau extra communautaire maghrébin est constitué dans le pays d'origine de personnes-ressources œuvrant au sein des délégations du Québec ou dans les ambassades canadiennes. Contrairement au réseau intra communau-

8. Le Canada et le Québec organisent à l'étranger diverses activités de promotion et, afin d'accroître leur attraction, cherchent également à véhiculer, grâce à des médias et/ou à des agences consulaires, l'image de pays au niveau de vie élevé, à l'activité économique florissante, respectueux de leurs citoyens, ouverts à l'immigration, valorisant la diversité culturelle et le dialogue interculturel (Chrétien 2003; Gouvernement du Canada 2005a; 2005b; Coderre 2003) 
taire, le réseau extra communautaire joue un rôle important dans la décision d'émigrer. De fait, il constitue souvent le premier contact concret de l'émigrant avec son futur pays de résidence. Malheureusement, les informations obtenues et/ou comprises lors de ce premier contact dans le pays d'origine sont souvent contredites par la réalité, notamment lors des premières étapes d'installation. Voici ce que dit un des interviewés ayant fait affaire avec un avocat dans son pays d'origine :

C'est lui [l'avocat], parfois il essaie par toutes ces possibilités d'attirer les clients. Des fois [il fait] même des facilités de paiement. Il te dit : «Voilà, si tu arrives au Canada, tu trouves des problèmes à trouver le logement, appelle-moi. Je connais du monde là-bas, ils peuvent trouver un logement». C'est ça, donc moi si j'allais par exemple dans un pays européen, je n'ai pas trouvé cette porte-là. Parce que nous, on fréquente les gens qui allaient en Europe. On a un nombre qui est très important en Europe qui dit que la première fois, on trouve de la difficulté à trouver du logement, à trouver de l'emploi. Mais eux [les avocats] ils essaient de jouer sur ce point-là. (migrant \#4)

Bien qu'il soit difficile d'étudier avec précision le travail d'information et de préparation fait en amont de la migration, il semble que ce travail donne l'impression aux candidats qu'une fois au Québec ils seront pris en charge, tout au moins durant les premiers mois de leur installation. Or, le fait de devoir faire face à des obstacles souvent inattendus sans l'aide directe des instances publiques amplifie les déceptions. À ce sujet, les intervenants rencontrés signalent que les candidats à l'immigration seraient peu enclins à écouter les mises en garde des personnesressources. Sans préjuger de la responsabilité des uns ou des autres, le discours des intervenants indique que le décalage entre les attentes et l'expérience migratoire ne peut qu'affecter négativement le processus d'insertion des immigrants maghrébins.

Si le capital humain des candidats à l'immigration est à l'origine de leur sélection, le réseau extra communautaire en situation pré migratoire devrait, quant à lui, servir à concrétiser la décision d'émigrer tout en exposant le plus largement possible les éléments positifs comme négatifs de l'immigration. Or, tout indique que ces réseaux de liens faibles contribuent à entretenir la méconnaissance des candidats de la réalité de la migration et des contextes culturel et socio-économique qui la conditionnent. Il semble y avoir une distorsion de la communication entre les candidats et les personnes-ressources. De plus, existent des intérêts personnels et institutionnels parfois antinomiques qui contribuent à accroître le niveau de déception des Maghrébins. ${ }^{9}$ Bref, à l'instar des liens

9. En effet, les logiques institutionnelles qui tendent à instrumentaliser l'immigration viennent fréquemment heurter les ambitions personnelles et professionnelles des migrants ainsi que l'ont montré Lenoir et al. (2008). 
faibles, les liens forts en situation pré migratoire sont d'une certaine utilité dans la mesure où ils permettent de consolider la décision d'émigrer, mais demeurent peu efficaces lorsqu'il s'agit d'une préparation adéquate à la réalité à laquelle sera confronté le nouvel arrivant.

Maintenant que nous avons vu l'impact des liens faibles et des liens forts sur la décision d'immigrer au Québec, nous verrons comment se constituent au fil du temps les réseaux en situation post migratoire. Nous verrons notamment les difficultés récurrentes des personnes à consolider des liens faibles pour obtenir une insertion professionnelle en accord avec leurs aspirations.

\section{RÉSEAUX POST MIGRATOIRES}

\subsection{Les difficiles premiers pas de la constitution de réseaux professionnellement porteurs}

Dans le contexte post migratoire ces réseaux de liens forts jouent également un rôle déterminant dans le processus d'installation :

Oui, on avait un ami qui était déjà ici au Québec, à Sherbrooke exactement. C'est pour ça qu'on a choisi Sherbrooke. Parce qu'on connaît le Québec, il y a plusieurs villes. On peut choisir l'une par rapport à l'autre (Montréal ou Sherbrooke) ... il faut s'informer. Et comme on avait un ami qui était installé ici, il nous a parlé de la ville, il nous a vraiment impressionné.... Donc, au fond, on a eu toutes ces informations. (migrant \#1)

Non seulement le réseau post migratoire, lorsque présent, facilite-t-il l'installation au Québec, mais il permet également une participation plus rapide aux programmes d'insertion et de mesures en emploi dispensés par le ministère de l'immigration du Québec ou ceux des centres locaux d'emplois :

Enfin, c'est l'ami que j'ai qui m'a.... Il m'a dit que voilà, il faut aller au Centre local d'emploi au cas où, par exemple on y trouve un agent et tout ça. Chose que j'ai faite. J'ai été au Centre local d'emploi. (migrant \#2)

Certains agissent même comme personnes-ressources auprès d'autres nouveaux arrivants:

Oui, c'est moi qui lui ai montré la place. Après son arrivée ici. À peu près 15 jours comme ça, j'ai dit : «c'est une bonne place pour apprendre à faire un $\mathrm{CV}$, connaître un peu le monde et parler, communiquer». (migrant \#4)

En retour, bien que ces formations aient pour objectif de créer des opportunités sur le marché du travail via certaines activités (foires de l'emploi, cocktail à la Chambre de commerce, rencontre avec des entrepreneurs, 
etc.), elles permettent surtout d'élargir les réseaux de liens forts. Les interviewés y développant plus souvent qu'autrement des liens d'amitié avec des personnes issues du même pays, voire de la même région ou de la même ville plutôt que des liens professionnels. Le fait que les formations permettent d'étendre le réseau post migratoire d'amis et de connaissances est perçu comme l'un des aspects les plus intéressants de ces formations.

L'élargissement du réseau de liens forts favorise une meilleure compréhension de sa propre trajectoire migratoire. Pour le dire autrement, ces nouveaux contacts agissent comme un miroir et favorisent une meilleure capacité réflexive face à sa propre condition. Ce type de réseau ne favorise toutefois pas l'obtention d'un emploi à court terme puisque les personnes rencontrées sont aussi en quête d'emploi. Dans un sens, cela va à l'encontre de certains objectifs du gouvernement quant à l'insertion professionnelle et l'accroissement des connaissances du contexte québécois (l'histoire, la culture, les valeurs et de leurs influences sur le fonctionnement des relations interpersonnelles et les normes propres au marché du travail local). ${ }^{10}$ En somme, l'accroissement et l'approfondissement des liens forts lors d'une participation aux ateliers de recherche d'emploi semblent avoir peu d'impact positif sur l'obtention d'un futur emploi au cours des premières années, et ce parce qu'elle ne favorise pas le développement d'un réseau professionnel. De fait, si la fréquentation des divers programmes gouvernementaux jouxtés à l'aide que peuvent fournir les non moins nombreux organismes d'aide aux nouveaux arrivants, sont des éléments facilitant l'installation à Montréal et Sherbrooke, une forte majorité des répondants a fait part de son scepticisme à l'égard du type de soutien qu'elle y obtient. La citation suivante reflète ce pessimisme :

Des amis m'ont dit qu'il n'y avait pas d'emploi dans l'informatique alors j'ai reculé un peu, donc je n'ai pas entrepris des démarches (de trouver un emploi dans le domaine de l'informatique). [à la question si la participation aux ateliers ont confirmé ces dires] Je dirais à $80 \%$ c'est confirmé, $20 \%$ il y a toujours un peu ... qui cherche. Ben, c'est sûr qu'il trouvera même si c'est saturé l'informatique. (migrant \#6)

Ainsi, la relation réseau intra/formation est relativement bien structurée et cela est confirmé par un intervenant (\#2) qui mentionne que «plus de gens connaissent déjà quelqu'un qui est ici, si la personne a

10. Notons que pour Emploi-Québec, l'intervention a pour objectif principal de favoriser l'intégration en emploi des nouveaux arrivants, alors que pour le Ministère de l'immigration et des communautés culturelles il s'agit de favoriser l'intégration à la société. Pour plus de détails sur leurs conceptions respectives de l'intégration il faut consulter leurs sites Internet : www.micc.gouv.qc.ca ; www.emploi-quebec.gouv.qc.ca 
déjà suivi (les formations) elle va l'encourager à le faire». Ce n'est pas que les formations soient jugées inutiles par les Maghrébins, mais tous les répondants s'entendent pour considérer ces formations comme des compléments à la recherche d'un emploi et non des moyens privilégiés d'insertion professionnelle et dans le but d'accroître davantage leurs réseaux de liens faibles, certains fréquentent les lieux de culte.

Bien que la majorité ne soit pas composée de fervents pratiquants, les lieux de cultes ont permis à certains d'accéder à une première forme d'intégration au Québec et d'amortir le choc culturel vécu. En ce sens, la mosquée devient un lieu de rassemblement qui favorise les relations humaines. Certains ont expliqué que c'est leur isolement au sein de la société québécoise qui les a poussés peu à peu à fréquenter la mosquée. D'autres vont plutôt considérer négativement les lieux de culte :

Personnellement moi j'ai horreur qu'une personne qui est musulmane, maghrébine qui vient ici, qui impose ses idées religieuses de manière fanatique, moi j' ai horreur de ça. On connaît un petit peu (le réseau religieux) quant on va à Montréal, ce n'est pas pour critiquer mes frères, mais il y a un certain constat qu'à certain moment, ça fait craindre. (migrant \#3)

C'est comme s'ils contribuent à salir toujours l'image de l'Islam. L'image des Maghrébins, parce que comme on dit, c'est toujours les bons qui paient pour les mauvais. Moi personnellement j'ai vu des personnes qu'on a rencontrées, des Maghrébins qui sont à Montréal. Qu'est-ce qu'ils font? Ils sont sur l'aide sociale. Ils restent sur l'aide sociale. Ils sont toujours à la mosquée en gardant la barbe, etc. Le Seigneur Dieu n'a jamais dit de vivre comme un parasite. (migrant \#11)

Critique acerbe si elle en est une, cette dernière citation n'indique pas une position négative à l'égard de la religion mais plutôt à l'endroit de ceux et celles qui fréquentent assidûment la mosquée et qui sont sans emploi. C'est comme si la mosquée devenait le lieu de rassemblement des personnes qui vivent des difficultés d'insertion professionnelle. Pour un interviewé, il y aurait un lien entre la présence d'un réseau de liens faibles et la baisse de fréquentation d'un lieu de culte:

Personnellement, entre autres à cause de tout ce qui s'est passé le 11 septembre et tout ça ... ça peut devenir trop dangereux de fréquenter la mosquée même si en vérité c'est quelque chose de personnel ... ce qui fait que personnellement, je pense qu'en Ontario, c'est parce que c'était ma famille (la mosquée), j'avais pas d'autre famille, or ici j'ai de la famille, j'ai des références, j'ai des devoirs vis-à-vis d'eux, de faire des choses ensemble et j'ai des amis. Mais c'est un changement de stratégie (de moins fréquenter la mosquée). (migrant \#3)

On remarquera le lien entre les événements du 11 septembre 2001 et les problèmes découlant de la fréquentation des lieux de culte. Quoi qu'il 
en soit, la présence à la mosquée n'est pas perçue comme une condition sine qua non de l'insertion mais comme une alternative pour consolider des relations dans un contexte où les réseaux sociaux sont peu développés. La fréquentation de la mosquée est pour certains un passage obligé vers une socialisation mais nullement une aide dans leur recherche d'emploi puisque cela ne permet pas d'élargir les réseaux et d'accéder à de l'information privilégiée sur d'éventuels emplois.

En somme, en situation post migratoire le réseau intra communautaire ne constitue pas un moteur d'accès à l'emploi. Il permet par contre de donner un sens à la recherche d'emploi, soit par l'effet miroir mentionné, soit parce que c'est à travers ces réseaux informels que se constitue peu à peu l'idée d'une communauté maghrébine :

Je vais dire une chose : la communauté maghrébine, c'est une communauté jeune ici. Elle n'est pas là depuis longtemps ici. La majorité vient d'arriver. Tous les jeunes que je connais sont en recherche d'emploi ou sont dans la période des études. Ils ont le même problème que moi. Parfois, au niveau de l'emploi, je reçois des offres de mes amis. Ils me disent : «J'ai vu un emploi comme ça, dans tel journal. Allez la voir». (migrant \#4)

L'image de la communauté maghrébine véhiculée parmi les nouveaux arrivants se situe entre une vision positive et négative; positive parce que créatrice de relations sociales et servant de zone mitoyenne entre le pays d'origine et la société d'immigration, négative parce que peu porteuse de rencontres permettant d'accéder à un premier emploi jugé convenable. Mentionnons que pour plusieurs le réseau formé de Maghrébins est constitué de relations qualifiées par eux-mêmes de «superficielles» et peu stables à moyen terme et est jugé fragilisé par le statut précaire des individus qui le composent (sans emploi, nouvel arrivant, etc.). Cette position ambiguë à l'égard de la communauté fait en sorte que les Maghrébins interrogés comptent davantage sur les canaux officiels (Centre locaux d'emploi, gouvernement, organismes communautaires) que sur leurs amis ou famille pour s'établir au Québec:

Non, il m'a pas hébergé. C'est-à-dire, j'ai eu des contacts avec lui pour me trouver un logement. Donc, il m'a aidé quand même à trouver. Donc, moi je suis devenu locataire dès le premier jour. C'est lui qui m'a montré la première semaine, les adresses. Après, je me suis débrouillée toute seule. (migrant \#2)

Une autre personne fait part de son isolement mais en rejetant le soutien du réseau maghrébin : «les contacts, par exemple, comme on dit nous : Tu es le fils de mon ami, tu peux travailler. Je ne suis pas comme ça, je ne connais pas » (migrant \#4). Ces deux passages tendent à valider l'idée selon laquelle les Maghrébins rencontrés valorisent davantage les 
stratégies individuelles que collectives pour leur insertion professionnelle. Cependant, le réseau extra communautaire n'en est pas moins source de déception pour les principaux intéressés.

\subsection{La poursuite du dialogue avec les institutions et le groupe majori- taire : vers une pérennité des distorsions?}

Tout réseau extra communautaire implique des relations entre les personnes récemment arrivés et le groupe majoritaire au Québec. Pour les Maghrébins, leurs contacts avec la majorité québécoise d'origine canadienne française tiennent à des relations professionnelles (lors de formation ou d'entrevues d'emplois) et formelles (lors de transactions économiques dans la vie quotidienne par exemple), mais qui ne favorisent pas le développement de liens faibles.

J'ai essayé de communiquer un petit peu avec des Québécois, je n'arrive pas parce que c'est des gens qui sont un petit peu froids. Peut-être c'est le climat. Ils sont rudes et timides. Ils lisent tout le temps dans le métro. (Migrant \#19)

\section{Ce phénomène est corroboré par un intervenant :}

Ils n'ont pas beaucoup d'amis québécois. Ils se tiennent vraiment en communauté avec d'autres cultures. Ça je m'aperçois de ça dans notre formation. Les gens, c'est étonnant comment ils vont aller vers les autres cultures aussi. Mais avec les Québécois, ils ont de la difficulté. (intervenant \#2)

Par contre, bien que l'on sente une certaine «conscience minoritaire» parmi ces nouveaux arrivants, il y a un désir d'insertion non seulement via l'emploi mais également sur le plan identitaire :

Parce que moi j'estime que je suis Québécois autant que toi. Même si t'es pure laine Québécoise...ce qui change, c'est juste les générations.... C'est juste une différence de génération de 200 ans, 300 ans. (migrant \#11)

Dans un sens, ce désir de participer à l'évolution de l'identité québécoise s'inscrit dans une volonté de briser certaines barrières qui sont autant de freins à l'insertion pleine et entière :

Donc, je découvre des choses qui me fascinent encore. Non seulement je dis « je viens, je respecte les valeurs québécoises tout en contribuant avec mes propres valeurs». Parce qu'à un certain moment, il y a toujours une forme de brassage, les cultures qui s'embrassent entre elles. C'est toujours pour arriver à donner quelque chose de meilleur. (migrant \#11)

Bien que cela constitue un phénomène généralisable à l'ensemble des personnes interrogées, on remarque une distinction entre les villes de Sherbrooke et de Montréal. Les Maghrébins installés à Sherbrooke 
semblent avoir une vision plus positive de leurs relations avec les Québécois d'origine canadienne française qu'à Montréal où peu d'entre eux fréquentent des personnes issues de ce groupe majoritaire. Une personne d'origine kabyle résume cette perception plus positive des personnes installées à Sherbrooke de leurs rapports avec les Québécois issus du groupe canadien français :

Juste pour dire que finalement ma venue ici n'est pas regrettable bien au contraire. En tant que 'Kabycois', je suis vraiment très fier de pouvoir m'intégrer comme je peux selon mes aspirations. C'est sûr avec parfois des petits défauts, des confrontations de choc culturels.... Mais toujours est-il, c'est vraiment très intéressant de pouvoir y contribuer, parce que je sais qu'il y a vraiment du travail avec ces maudits Québécois! (migrant \#7)

Tout d'abord, le terme 'Kabycois' pour s'auto désigner marque une volonté de s'intégrer à une identité québécoise tout en conservant l'identité d'origine. Ici, on rejoint le passage cité plus haut sur la volonté de faire évoluer l'identité québécoise et où la distance entre le Eux et le Nous est atténuée par cette identité hybride. En ce qui concerne l'identité kabyle, on remarquera que l'identité algérienne, puisque les Kabyles ont la citoyenneté algérienne, est à toute fin pratique occultée au sein de cette 'kabycoisité' (l'expression est de nous). Quoiqu'il en soit, les relations entre majoritaires et nouveaux arrivants maghrébins, principalement à Montréal, se limitent à des relations d'ordre professionnel lors des diverses formations que suivent les Maghrébins tout au long de leur cheminement et où ils sont appelés à rencontrer des employeurs.

Outre le peu de relations avec les Québécois d'origine canadienne française, les immigrants mentionnent d'autres barrières à l'emploi telles que l'accessibilité aux ordres professionnels, la connaissance de l'anglais exigée par les employeurs et le manque d'ouverture de la société québécoise à l'égard des nouveaux arrivants. Autant de sources de déception dont l'intensité varie selon les personnes et leurs attentes lors de leur arrivée au Québec mais qui est ressentie par l'ensemble des nouveaux arrivants :

Oui, on te laisse ... à minuit, on te laisse. Tu descends de l'aéroport, tu ne connais personne et c'est vraiment difficile. Quand on est arrivé, une semaine après, j'ai dit à ma femme : 'Sois courageuse, on va repartir dans notre pays'.... Mais sinon, pour mes enfants, ils sont super bien, ça leur plaît beaucoup. (migrant \#6)

Bien que généralisée, cette déception face aux services d'accueil et d'intégration est tributaire du contexte socioculturel d'où proviennent les immigrants. Il appert, selon les intervenants, que les personnes originaires de l'Algérie s'appuieraient davantage sur l'État pour les aider dans 
leurs démarches d'installation et de recherche d'emploi que les autres Maghrébins. Rien dans les analyses effectuées ne permet de vérifier ces affirmations mais on peut supposer que l'interventionnisme d'État en Algérie, de tendance socialisante, favoriserait chez les citoyens des attentes plus grandes envers l'État, attentes transposées en contexte migratoire. ${ }^{11}$

Pour revenir à la distinction entre les deux contextes d'insertion, on note des similarités et distinctions. Tant à Montréal qu'à Sherbrooke il y a présence d'associations au service des personnes nées à l'étranger. Toutefois, à Sherbrooke il semble y avoir de meilleures possibilités pour les Maghrébins de développer des réseaux extra communautaires. Cela explique en partie l'attitude plus positive des personnes installées à Sherbrooke à l'égard de la société québécoise et de leur place au sein dans cette société. ${ }^{12}$ À l'opposé, dans le contexte montréalais on note un certain découragement de la part des Maghrébins rencontrés :

Oui, un ami qui travaille là-bas (à Sherbrooke).... Qui m'a dit : «Venez à Sherbrooke, vous serez mieux installés par rapport à la ville». Parce qu'à la ville, effectivement pour nous déjà, quand on est arrivé, c'était difficile parce qu'on était pas habitué à une grandeur comme ça.... C'est un choc pour nous mais on est venu. Je suis resté à Montréal parce que j'ai trouvé des gens de notre région. Pour ne pas déchirer directement de notre pays. Peut-être pour quelques temps et je pense partir ailleurs. (migrant \#6)

Ce sentiment de découragement semble s'accroître lorsque les interviewés fréquentent d'autres Maghrébins qui n'ont pas réussi à dénicher un emploi dans leur domaine en dépit de leur présence plus longue à Montréal. Lorsque leurs connaissances réussissent à trouver un emploi dans leur secteur, les Maghrébins rencontrés affirment vouloir couper les ponts avec ces connaissances par crainte d'être désillusionnés face à leur propre recherche d'emploi. C'est dire que les liens faibles peuvent doublement accentuer l'effet de découragement en ne permettant pas d'obtenir des informations pertinentes ou encore en stratifiant les statuts entre personnes de même origine. En somme, tout se passe comme si les Maghrébins rencontrés craignent de ne pouvoir réussir leur projet migratoire et de sombrer dans une déprime similaire à celle de ceux qui sont toujours sans emploi. Dans ce cas, le réseau ethnique ne favorise pas l'accès à un premier emploi mais créé un découragement qui nuit à

11. Pour une analyse plus détaillée des entrevues réalisées auprès d'intervenant en recherche d'emploi, voir op. cit. Lenoir et al. (2008).

12. Ceci pourrait s'expliquer en partie par le fait que Sherbrooke, tout en demeurant une ville de petite taille, a reçu un nombre significatif, sur une dizaine d'années, d'immigrants et de réfugiés d'origine diverses (Colombie, Croatie, Serbie, Maghreb, etc.). Cela pourrait avoir un effet positif sur les relations entre communautés ethnoculturelles et surtout sur une plus grande personnalisation des services offerts aux nouveaux arrivants. 
la solidarité communautaire, voire à la constitution d'une communauté d'histoire et de culture en contexte migratoire.

\section{Conclusion}

Au cours des pages précédentes, nous avons vu que la question des réseaux sociaux s'inscrit directement au sein d'une dynamique complexe impliquant plusieurs acteurs individuels, collectifs et institutionnels. À ce titre, l'objectif de cet article n'était pas d'établir des solutions concrètes aux problèmes d'insertion professionnelle vécus par les nouveaux arrivants, mais plutôt de contribuer à la connaissance empirique de cette thématique pour en avoir une meilleure compréhension. L'appel à la sociologie des réseaux sociaux a permis de faire ressortir les difficultés pour les Maghrébins de notre échantillon à accéder à l'information pertinente quant aux emplois qualifiés disponibles. La portée de leurs réseaux étant à la fois restreinte par les conditions dans lesquelles l'insertion socio-économique de cette population se fait (haut taux de chômage, déqualification), et trop large parce que les liens tissés lors des premières années d'installation sont fragiles. Comme les Maghrébins interrogés sont tous arrivés au Québec dans la foulée des attentats du 11 septembre 2001, il est plausible que ces évènements jouent en leur défaveur lors de leur recherche d'emploi. Malgré tout, notre étude ne repose pas sur une comparaison pré et post 11 septembre 2001 c'est pourquoi rien ne nous permet d'extrapoler sur cette question. La revue de littérature présentée dans la deuxième section permet de faire ressortir le caractère original de notre échantillon (immigrants récents d'origine maghrébine, travailleurs qualifiés, installation dans une ville à haut niveau de diversité ethnoculturelle et une ville plus homogène) par rapport à ce qui a été fait tout en l'inscrivant dans un courant théorique et empirique qui cherche à comprendre l'apport des réseaux sociaux à l'insertion des immigrants, toute catégorie confondue.

Quant aux propositions de départ, certaines ont pu être validées en tout ou en partie alors que d'autres apparaissent comme non pertinentes en fonction des données obtenues. À la proposition A, dans le cas spécifique des Maghrébins il ne semble pas y avoir de liens entre les réseaux et l'obtention d'un emploi. De plus, il appert que les trajectoires individuelles, si elles ont peu d'impact sur l'insertion à proprement parler, agissent néanmoins sur les perceptions des individus ce qui, en dernière instance, peut avoir une influence positive ou négative sur l'insertion à plus long terme. Dans le cas des Maghrébins interviewés, il fallait prendre en considération les réseaux présents au sein de la société d'origine puisque, plusieurs études récentes l'ont montré (Helly 1995; 
Lenoir 2006a; 2006b), on doit tenir compte de l'existence de diasporas et de communautés d'histoire et de culture constituées en contexte post migratoire. Quant à la deuxième proposition sur la présence et le travail des associations représentant des groupes ethnoculturels spécifiques, rien n'indique que les personnes interviewées fassent appel à ces organisations dans leur quête d'un emploi. Cela montre jusqu'à un certain point les difficultés de ces associations à faciliter l'insertion économique des Maghrébins interrogées. Quant aux organismes gouvernementaux et paragouvernementaux, la proposition $\mathrm{C}$, ils jouent un rôle important mais leur pouvoir dans l'élaboration de mesures pour contrer les difficultés rencontrées est limité. En ce qui concerne la proposition $\mathrm{D}$, notre étude ne permet pas de déterminer si l'obtention d'un premier emploi, même en dessous des qualifications possédées, favorise la mobilité socio-économique. Certaines personnes ont affirmé qu'un premier emploi leur avait redonné du courage et de la détermination mais puisque toutes les personnes interrogées étaient en quête d'emploi, nous ne saurions conclure sur cette question. Les résultats obtenus permettent de conclure que ce n'est pas le manque de réseaux sociaux qui constitue un problème majeurs des Maghrébins mais le peu d'impact de leurs réseaux pré et post migratoires. Les difficultés pour les institutions en place de favoriser le développement de liens faibles témoignent de la pluralité des obstacles et barrières auxquels sont confrontées les personnes que nous avons interrogées. De même, le peu de considération de la société québécoise à l'égard des compétences obtenues dans le pays d'origine alimentent les problèmes systémiques vécus par cette population. En cela, toute généralisation des résultats doit être mise en contexte en fonction de ce lien entre pays d'origine et société d'immigration (Schéma 1). En dépit de la complexité de la problématique étudiée, certaines conclusions peuvent être tirées.

Dans un premier temps, une stratégie individualiste est perceptible lors de la recherche d'emploi. S'apparentant à un mécanisme de défense pour ne pas répéter les erreurs faites par les membres de la communauté, la recherche d'emploi emprunte des chemins sinueux nécessitant de constantes adaptations au nouvel environnement. Ici, la communauté ne sert pas tant de levier à l'insertion que de miroir, parfois déformant il est vrai, reflétant les potentialités et les barrières existantes. Sur ce point, la très grande majorité des interviewés a affirmé ne pas chercher à tout prix à entretenir des liens étroits avec la communauté d'origine si ce n'est que lors de rencontres sporadiques. À cet effet, tout indique que dans le cas des Maghrébins interviewés le groupe ethnique ne constitue pas une valeur sûre d'insertion et des recherches plus poussées en ce domaine permettraient certainement d'en comprendre les raisons. On constate même que dans certains cas il y a rejet de la communauté qui sert plutôt d'exemple à ne pas suivre au lieu d'être un modèle d'insertion. Les ré- 
seaux personnels se consolident plutôt autour de réseaux constitués par la recherche d'emploi notamment dans les centres d'emploi, voire dans la relation entre les intervenants et les chercheurs d'emploi. Là réside le problème car ce sont les liens faibles, donc moins chargés d'émotions et d'affect, qui devraient prédominer dans la relation avec les intervenants plutôt que des liens de proximité dont les individus attendent beaucoup. Cependant, les liens entretenus avec des membres de la communauté maghrébine peuvent avoir des effets bénéfiques à long terme mais notre étude ne permet pas d'en mesurer les effets sur une plus longue période de temps. Il est plausible, par exemple, que ces liens permettent une stabilisation émotionnelle qui, à son tour, aurait des effets ultérieurs positifs sur l'intégration au marché du travail. D'autres recherches sur cette question devraient être effectuées.

C'est parce que la relation entre les structures d'accueil (organismes gouvernementaux) aux immigrants et la clientèle se construit autour d'une certaine proximité que la participation aux diverses formations ne permet pas d'élargir les réseaux et de consolider les liens faibles. Il en va de même pour ce qui est des difficultés inhérentes à l'entrée dans les ordres professionnels. Même lorsque cela est possible, l'appartenance à un de ces ordres ne constitue pas une solution aux problèmes réels. Sur le plan pratique, le problème se situerait plus au niveau de l'accès au stage et de la relation entre les programmes d'insertion et les entreprises elles-mêmes qu'au niveau du nombre de formations et d'organismes existants. De plus, les liens entre organismes et entreprises sont peu formalisés et plusieurs ont affirmé que leurs rencontres se limitaient à un échange de cartes d'affaire sans résultats concrets. On voit qu'en dépit des efforts déployés par les instances gouvernementales et para gouvernementales, peu de programmes favorisent un élargissement concret des réseaux de contacts.

Quant à la comparaison Montréal-Sherbrooke, on observe qu'un milieu multiculturel comme Montréal, perçu comme plus favorable à l'insertion, ne garantit pas nécessairement une insertion plus rapide. Ainsi, pour les Maghrébins la fréquentation des 'pairs' est une condition nécessaire à l'adaptation à la nouvelle société mais non suffisante pour l'insertion professionnelle espérée avant le processus migratoire. À l'opposé, le contexte sherbrookois semble favoriser un élargissement plus rapide des réseaux sociaux. Cela pourrait s'expliquer par l'obligation, pour les Maghrébins, d'accroître rapidement leurs liens faibles compte tenu de la faiblesse du réseau communautaire maghrébin à Sherbrooke. Cet avantage pour les Maghrébins installés à Sherbrooke par rapport à ceux vivant à Montréal rejoint les conclusions d'une étude récente de Statistique Canada qui constate que les performances économiques des immigrants en région sont meilleures que celles de ceux habitant les grands centres 
urbains (Statistiques Canada 2008).$^{13}$ Le graphique suivant résume l'ensemble du processus de sélection et d'installation des nouveaux arrivants que nous avons abordé au cours des pages précédentes :

\section{Graphique 1 : Réseaux sociaux et contexte d'installation des nouveaux arrivants d'origine maghrébine}

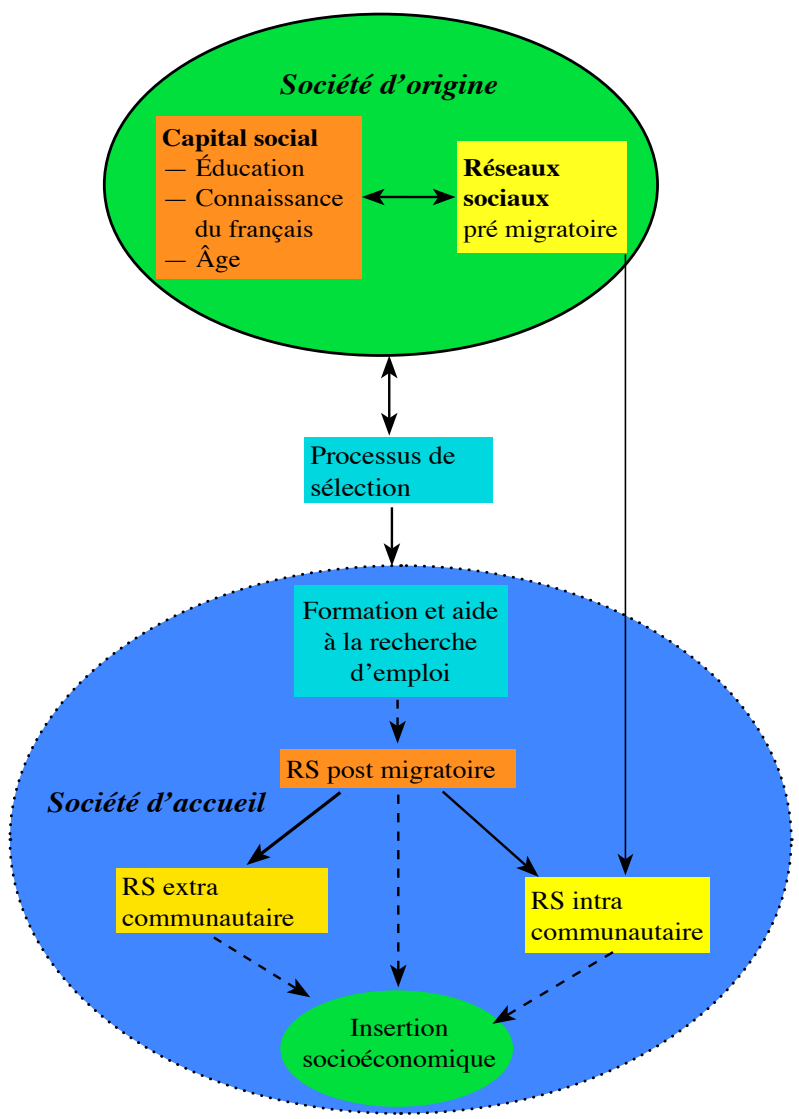

13. On observe des différences de genre qui méritaient d'être étudiées ultérieurement. Les femmes auraient accès à des réseaux de liens forts et de liens faibles plus limités que les hommes. Cela vient corroborer des études précédentes (Campbell et Rosenfeld 1985; Moore 1990; Salaff et Greve 2004) mais à partir des données que nous avons obtenues les femmes, même si elles ont des réseaux plus fragiles et moins élargis, auraient plus de facilité à se trouver un emploi dans des secteurs autres que leur formation de départ. Cela s'explique en partie par leur plus grande disposition à accepter des emplois en dessous de leurs compétences. De plus, il y a lieu de penser que la flexibilité avec laquelle les femmes maghrébines articulent leur identité multipolaire puisse, comme le souligne Vatz-Laaroussi (2008), «leur permettre d'asseoir des liens, des solidarités et des stratégies fonctionnelles dans le contexte nouveau avec lequel elles ont à vivre avec leur famille et elles sont très critiques vis-à-vis des instances qui les catégorisent selon l'une ou l'autre seulement de ces références identitaires» (p. 21). 
Ce schéma permet de voir les possibilités pour les nouveaux arrivants de s'insérer sur le marché de l'emploi en fonction des réseaux constitués et du capital humain de chacun. Cela dit, le chemin vers cette insertion n'est pas linéaire et, comme l'indiquent les flèches pointillées dans le graphique, elle est constamment mise en danger lors de la trajectoire au sein de la société d'immigration. De même, on constate que c'est dans l'interrelation avec la société d'accueil que se trouve la clé de la compréhension des problèmes vécus. En somme, et pour répondre succinctement à la question de départ, les réseaux sociaux ne semblent pas jouer un rôle majeur dans l'insertion professionnelle des Maghrébins au cours des premières années d'installation. Ces réseaux sociaux peuvent néanmoins faciliter l'adaptation et les chocs culturels propres aux processus migratoires. Ainsi, les réseaux sociaux existants au sein des communautés maghrébines semblent conditionner les difficultés auxquels font face les individus en provenance de pays du Maghreb, et ce nonobstant leur durée de séjour à Montréal et Sherbrooke. Si les réseaux favorisaient l'ouverture aux liens faibles, il est permis de croire que les difficultés, sans être éliminées, seraient de moindre importance.

Les résultats obtenus permettent difficilement de généraliser à l'ensemble des nouveaux arrivants, ni à tous les Maghrébins par ailleurs. Cependant la méthode de collecte et d'analyse de données favorise la mise à jour des représentations que développent les personnes interrogées du long processus d'insertion socio-économique auquel ils sont confrontés. La codification effectuée aura permis de mettre en lien les aspirations des individus avec des éléments plus 'objectifs' tels que les compétences et les réseaux de contact qu'ils possèdent. De manière générale, tant l'approche par réseau social que la méthodologie qualitative employée s'inscrit dans une approche compréhensive qui, certes s'intéresse aux faits sociaux, mais met l'accent sur la construction des réseaux au sein de dynamiques migratoires et à la représentation qu'en font les acteurs.

\section{BibLIOGRAPHIE}

Amuedo-Dorantes, Catalina et Kusum Mundra. 2007. Social networks and their impact on the earnings of Mexican migrants. Demography 44(4):849863.

Andreani Jean-Claude et Françoise Conchon. 2003. Les méthodes d'évaluation de la validité des enquêtes qualitatives en marketing. Congrès des Tendances du Marketing (Novembre).

Bauder, Harald. 2001. Employment, ethnicity and metropolitan context: The case of young Canadian immigrants. Journal of International Migration 
and Integration/Revue de l'intégration et de la migration internationale 2(3):315-341.

Bergeron, Jean et Stéphanie Potter. 2006. Family members and relatives: An important resource for newcomers' settlement? Canadian Issues/Thèmes canadiens Printemps:76-80.

Bonacich, Edna. M. 1973. A theory of middlemen minorities. American Sociological Review 38(5):583-94. 1976. Advanced capitalism and Black/White race relations in the USA: A split labor market interpretation. American Sociological Review 41(1):34-51.

Boudon, Raymond. 1988. Individualisme ou holisme : un débat méthodologique fondamental. Dans Henri Mendras et Michel Verret, dir. Les champs de la sociologie française. Paris: Armand Collin.

Bourdieu, Pierre. 1980. Le capital social : notes provisoires. Actes de la recherche en sciences sociales 3:2-3.

Campbell, Karen et Rachel A. Rosenfeld. 1985. Job search and job mortality: Sex and race differences. Research in the Sociology of Work 3:147-174.

Cardinal, Lise (en collaboration avec Johanne Tremblay). 1998. Comment bâtir un réseau de contacts solide. Dans Fondation de l'entrepreneurship. Montréal: Les éditions Transcontinentales/Les éditions de la Fondation de l'entrepreneurship.

Chrétien, Jean. 2003. L'immigration et le multiculturalisme au Canada. Conférence présenté au Sommet de Londres sur la gouvernance progressiste, le 12 juillet 2003. http://www.pcobcp.gc.ca

Coderre, Denis. 2003. Rapport sur le rendement pour la période se terminant le 31 mars 2003. Secrétariat du Conseil du trésor Canada, Citoyenneté et Immigration Canada. http://www.tbs-sct.gc.ca

Coleman, James S. 1990. Foundations of Social Theory, Cambridge, Belknap.

Collyer, Michael. 2005. When do social networks fail to explain migration? Accounting for the movement of Algerians. Journal of Ethnic and Migration Studies 31(4):699-718.

D’Unrug, Marie-Christine. 1974. Analyse de contenu. Paris : Éditions Universitaires/encyclopédie universitaire.

Freeman, Linton C. 1979. Centrality in social networks: Conceptual clarification. Social Networks 1:215-239.

Frenette, Marc et René Morissette. 2003. Convergeront-ils un jour? Les gains des travailleurs immigrants et de ceux nés au Canada au cours des deux dernières décennies. Ottawa: Statistique Canada, « Direction des études analytiques : documents de recherche », no 215, 23 p.

Fuglerud, Oivind et Ada Engebrigtsen. 2006. Culture, networks and social capital: Tamil and Somali immigrants in Norway. Ethnic and Racial Studies 29(6):1118-1134. 
Galbraith, John Kenneth. 1971. The New Industrial State. New York: Mentor.

Garcia, Carlos. 2005. Buscando trabajo: Social networks among immigrants from Mexico to the United States. Hispanic Journal of Behavioral Sciences 27(1):3-22.

Gardin, Jean-Claude. 1974. Les analyses de discours. Neuchâtel : Delachaux et Niestlé, (Collection zéthos).

Gauthier, Benoît (sous la direction de). 2009. Recherche sociale : de la problématique à la collecte de données (5ième édition). Ste-Foy : Presses de l’Université du Québec.

Germain, Annick, Yvon Martineau, et Jaël Mongeau. 2001. Les caractéristiques sociodémographiques et socioéconomiques des résidants et de la clientèle des centre locaux d'emploi de l'île de Montréal : rapport et synthèse Direction régionale de Montréal. Emploi-Québec, INRS-Urbanisation, Culture et Société.

Gouvernement du Canada. 2000. (septembre) Résultats des immigrants sur le plan des compétences et rôle du capital humain spécifique à une région d'origine. Ottawa: Ressources humaines et développement social. www. rhdsc.gc.ca

2005a. Créer, connecter et partager les connaissances : dialogue sur les liens entre les collectivités. Ottawa: Infrastucture Canada : Recherche et analyse.

2005b. Bienvenue au Canada: Ce que vous devriez savoir. Ottawa: Citoyenneté et immigration Canada, no de catalogue. Ci-87/2003F.

Gouvernement du Québec. 2004a. Des valeurs partagées, des intérêts communs. Pour assurer la pleine participation des Québécois des communautés culturelles au développement du Québec. Plan d'action 2004-2007.

2004b. Ministère de l'immigration et des communautés culturelles. www.micc.gouv.qc.ca

2004c. Emploi-Québec. www.emploiquebec.net

Goza, Franklin. 2003. Social networks and the incorporation of Brazilians in Canada and the United States. (in Portuguese). Pp. 263-288 dans Ana Cristina Braga Martes and Soraya Fleischer, dir. Fronteiras Cruzadas: Etnicidade, família e redes sociais. São Paulo: Paz e Terra.

Granovetter, Mark. 1973. The strength of weak ties. American Journal of Sociology 78:1360-1380.

1985. Economic action and social structure: The problem of embeddedness. American Journal of Sociology 91(3): 481-510.

1990. The old and the new economic sociology. Dans R. Friedland et A.F. Robertson, dir., Beyond the Market Place, Rethinking Economy and Society. New York, Aldine de Gruyter.

Helly, Denise, Nong Zhu, et Marilou Trudel. 2008. Équité et insertion des immigrants au marché du travail canadien. Association européenne d'économie sociale, Congrès de Reims, 8-10 septembre 2008, À être publié. 
Helly, Denise (avec la collaboration de D. Tessier et N. van Schendel). 1995. Valeurs familiales et information gouvernementale : Perceptions d'immigrés. Québec : Institut québécois de recherche sur la culture.

Institut de la statistique du Québec. 2007. www.stat.gouv.qc.ca

Iosifides, Theodoros, Mari Lavrentiadou, Electra Petracou, et Antonios Kontis. 2007. Forms of social capital and the incorporation of Albanian immigrants in Greece. Journal of Ethnic and Migration Studies 33(8):13431361.

Kazemipur, Abdolmohammad. 2006. The market value of friendship: Social networks of immigrants. Canadian Ethnic Studies 38(2):47-71.

Lenoir-Achdjian, Annick. 2006a. Appréhender la nation, vivre la diaspora : regards arméniens. Bruxelles: Academia-Bruylant, Coll. Carrefour. 2006b. Éducation à la citoyenneté et contextes de diaspora : dilemmes d'appartenances multiples et stratégies d'allégeances concurrentielles. Dans C. Xypas, dir., Education à la citoyenneté et diversité culturelle : confrontation des modèles européens et nord-américains. Regards critiques sur les fondements conceptuels, les discours et les pratiques. Paris: PUF.

A. Lenoir-Achdjian, I. Drainville, D. Helly, M. Vatz-Laaroussi, S. Arcand, et A. Mahfoudh. 2008. The professional insertion of immigrants born in the Maghreb: challenges and impediments for intervention. Journal of International Migration and Integration 8(1). 21p.

Li, Peter S. 2004. Social capital and economic outcomes for immigrants and ethnic minorities. Journal of International Migration and Integration 5(2):171-190.

Lo, Lucia. 2003. Resources and immigrants economic integration. Présentée dans le cadre de la 6ième conférence nationale Metropolis, Edmonton, Alberta (Mars 2003).

Loury, Glen. 1977. A dynamic theory of racial income differences. In Phyllis A. Wallace, ed., Women, Minorities and Employment Discrimination. Lexington, MA: Lexington Books.

Mercklé, Pierre. 2004. Sociologie des réseaux sociaux. Paris: La Découverte, coll. 'Repères'.

Molina, José Luis et Christopher McCarty. 2007. Personal networks and ethnic identifications: The case of migrants in Spain. International Sociology 22(6):721-741.

Moore, Gwen. 1990. Structural determinants of men's and women's personal networks. American Sociological Review 55:726-735.

Mucchielli, Alex. 1996 Dictionnaire des méthodes qualitatives en sciences humaines et sociales. Paris: Armand Colin.

Paré, Sylvie. 2002 "Réseaux, réseautage ? Le rôle des associations chez les petits entrepreneurs ethniques montréalais." Texte préparé en vue de la Con- 
férence de l'Association Internationale de Recherche en Entrepreneuriat et en PME, HEC Montréal.

Piché, Victor, Jean Renaud, et Lucie Gingras. 2002. L'insertion socio-économique des nouveaux immigrants sur le marché du travail : une approche longitudinale. Population 57(1):63-90.

Picot, G. 2004. The deteriorating economic welfare of Canadian immigrants. Canadian Journal of Urban Research 13(1):25-46.

Portes, Alejandro et Robert D. Manning. 1986. The immigrant enclave: Theory and empirical examples. Pp. 47-68 dans Olzak et Nagel, dir., Competitive Ethnic Relations. Orlando, FL: Academic Press, Inc./Harcourt Brace Jovanovich.

Potocky-Tripodi, Miriam. 2004. The role of social capital in immigrant and refugee economic adaptation. Journal of Social Service Research 31(1):5989.

Préfontaine, Jean-Paul et Andrew Benson. 1999. Barrier to Canadian immigrants' economic integration: Government response to market failure. Vancouver: Immigration Policy, Planning and Research Division, Ministry responsible for Multiculturalism, Human Rights and Immigration, Government of British Columbia, 3ième conference Métropolis (janvier 1999).

Putnam, Robert D. 2001. Bowling Alone: The Collapse and Revival of American Community. New York: Simon and Schuster.

2007. E pluribus unum: Diversity and community in the twenty-first century. Scandinavian Political Studies 30(2):174.

Reitz, Jeffrey G. 2005. Tapping immigrants' skills: New directions for Canadian immigration policy in the knowledge economy. Choices 11(1):1-18.

Renaud, Jean, Victor Piché, et Jean-François Godin. 2003. L'origine nationale et l'insertion économique des immigrants au cours de leur dix premières années au Québec. Sociologie et Sociétés 35(1):165-184.

Salaff, Janet W. et Arent Greve. 2004. Can women's social networks migrate? Women's Studies International Forum 27:149-162.

Statistique Canada. 2004. Immigration et citoyenneté, Recensement de 2001 Statut d'immigrant et lieux de naissance du répondant, sexe et groupes d'âge pour la population. Ottawa: Catalogue 95F0357XCB2001009. 2005. Enquête longitudinale auprès des immigrants du Canada : S'établir dans un nouveau pays : un portrait des premières expériences. Ottawa: Catalogue no. 89-614-XIF.

2006. Étude : Les immigrants sur le marché canadien du travail en 2006 : analyse selon la région ou le pays de naissance. Ottawa.

2008. Les immigrants dans les régions. Perspective No. 75-001-X : 5-16.

Sweetman, Arthur. 2006. Need we pursue immigration objectives one at a time? Economic growth, family reunification and points systems. Thèmes canadiens/Canadian Issues, printemps: 68-71. 
Vatz-Laaroussi, Michèle. 2008. Les femmes maghrébines immigrantes au Québec : insertion en emploi, déqualification professionnelle et dynamiques familiales. À être publié dans Travail Genre et Société.

Wilson, L. Kenneth et Alejandro Portes. 1980. Immigrant enclaves: An analysis of the labor market experiences of Cubans in Miami. American Journal of Sociology 86(2):295-319.

Woolcock, Micheal. 2001. The place of social capital in understanding social and economic outcomes. Isuma-Revue canadienne de recherche sur les politiques 2(1), Printemps.

Sebastien Arcand, Ph.D. Sociology, is an assistant professor at Hautes études commerciales in Montreal (HEC Montreal). He is the coordinator of the domain 2: economic integration/labor market integration at Quebec Metropolis CentreImmigration and Metropoles. His works are related to cross-cultural management, ethnic entrepreneurship, integration of minorities to labor market and sociology's contribution to organizational studies. He publishes in English, Spanish, and French and teaches in Colombia, Latin America.

Annick Lenoir-Achdjian, Ph.D. in Anthropology, is a professor at the Department of social work at the Université de Sherbrooke. Her expertise concerns the understanding of migratory process and the establishment of immigrant families as well as intervention and the effects on these families. She is the author of several publications, research studies, reports and articles, including notably Appréhender la nation, vivre la diaspora : regards arméniens. Coll. Carrefour, Bruxelles; Academia-Bruylant, 2006. From 2003 to 2007, Annick Lenoir-Achdjian coordinated the Demographic and Demo-Linguistic Aspects of Immigration component at the Immigration and Metropolis immigration centre. She is now acting coordinator at Quebec Metropolis Centre-Immigration and metropoles of the emerging Domain "Welcoming Communities" with Michèle Vatz Laaroussi.

Denise Helly is a senior researcher at Institut National de Recherche Scientifique. She was trained in anthropology (PH.D La Sorbonne, 1975), sociology, political science, and sinology and specialized in studies on ethnic minorities, citizenship, nationalism, policies of cultural pluralism and of immigration, discrimination, Islam in the West, and State and religion regimes. She has made several surveys on integration of immigrants in Quebec, published 10 books and numerous articles covering topics such as Chinese overseas, national minorities in China, Chinese in Canada, Canadian multiculturalism, history of the ideas of citizenship and nation, Québec policy towards cultural minorities, and Muslims in Canada and Europe. 